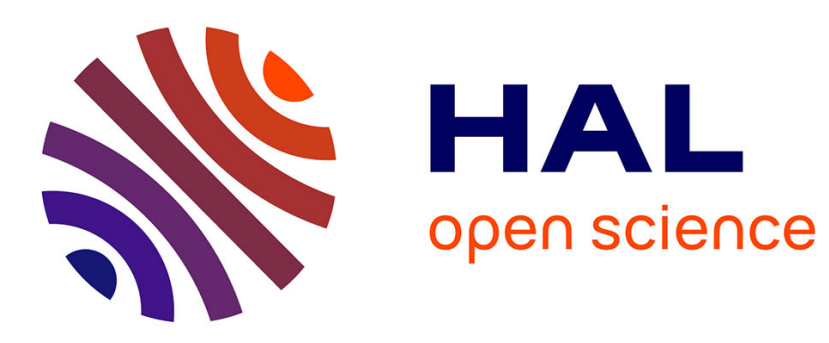

\title{
InPhysible: Camouflage Against Video-Based Physiological Measurement
}

Daniel Mcduff, Christophe Hurter

\section{To cite this version:}

Daniel Mcduff, Christophe Hurter. InPhysible: Camouflage Against Video-Based Physiological Measurement. EMBC 2018, 40th International Engineering in Medicine and Biology Conference, Jul 2018, Honolulu, United States. pp.5784-5789, 10.1109/EMBC.2018.8513662 . hal-02917102

\section{HAL Id: hal-02917102 \\ https://hal-enac.archives-ouvertes.fr/hal-02917102}

Submitted on 18 Aug 2020

HAL is a multi-disciplinary open access archive for the deposit and dissemination of scientific research documents, whether they are published or not. The documents may come from teaching and research institutions in France or abroad, or from public or private research centers.
L'archive ouverte pluridisciplinaire HAL, est destinée au dépôt et à la diffusion de documents scientifiques de niveau recherche, publiés ou non, émanant des établissements d'enseignement et de recherche français ou étrangers, des laboratoires publics ou privés. 


\title{
InPhysible: Camouflage Against Video-Based Physiological Measurement
}

\author{
Daniel McDuff ${ }^{1}$ and Christophe Hurter ${ }^{2}$
}

\begin{abstract}
Imaging photoplethysmography (iPPG) is a powerful set of methods for measuring physiological signals from video. Recent advances have shown that a low-cost webcam can be used to measure heart rate, blood flow, respiration, blood oxygen levels and stress. While these methods have many beneficial applications, the unobtrusive and ubiquitous nature of the sensors risk exposing people to unwanted measurement. We present InPhysible the first camouflage system against videobased physiological measurement. The infra-red system can be embedded into any pair of glasses, or other headwear, and disrupts the measurement of the iPPG signal while being imperceptible by the human eye. Our system is flexible and can simulate realistic pulse signals to hinder heart rate measurement. In this paper we present the design of our prototype and a user study validating its efficacy. Finally, we discuss the limitations and implications for data privacy and security.
\end{abstract}

\section{INTRODUCTION}

Video-based physiological measurement has advantages over contact sensors, perhaps the most significant being that ubiquitous low-cost cameras can be used, negating the need for customized hardware [1], [2]. In addition, the vital signs of multiple people can be measured concomitantly using the same sensor [3] and head-worn cameras can capture this signal even in the presence of motion [4], [5], [6]. Videobased methods can be used to measure the blood volume pulse [3], heart rate (HR), respiration, heart rate variability (HRV) and blood oxygenation [7]. Blood flow can also be spatially visualized on the skin [8], [5].

However, the fact that these imaging techniques are unobtrusive and low-cost presents problems. It is possible to instrument a space or design a head-worn camera that allows the physiological parameters of other person to be measured. How can someone opt-out of such measurement? What are the optimal properties of a system that interferes with iPPG? Can we design a system that does not result in social stigma? We surveyed 76 people who used a remote physiological visualization tool that allowed them to "see" the HR and blood flow of a person they were interacting with. Of these people 53\% said that knowing someone could measure their vital signs with a webcam would make them nervous during social interactions. However, $65 \%$ of people said they would be willing to use the system to measure the physiological signals of other people in that context.

When applied in-situ, iPPG technology can be used to perform non-explicit monitoring of people who have not

\footnotetext{
${ }^{1}$ Daniel McDuff is a Researcher at Microsoft Research, Redmond, USA. damcduffemicrosoft.com

${ }^{2}$ Christophe Hurter is a Professor at Ecole Nationale de l'Aviation Civile, University of Toulouse, France. christophe.hurterdenac.fr
}

consented and are not aware that they are being recorded. We see a necessity to develop an antidote, a way to prevent this monitoring. There is a need for a system that hinders pulse wave retrieval. A device that emits an electromagnetic signal could be used to disrupt iPPG measurements. If such a device existed an iPPG method might struggle to distinguish between the actual heart beat and a spoof signal. We propose to use infra-red (IR) light, precisely because it will be invisible to the unaided eye and typically effects the measurements of RGB cameras. However, other types of illuminations could also result in interference.

We present InPhysible, a wearable device that camouflages the wearer from video-based remote physiological measurement. Figure 1 shows a schematic of the system and an example of the impact on the recovered pulse measurement. In the following paper, we: 1) describe the hardware and software design; 2) present a user study validating the effectiveness of the device in obstructing remote physiological measurements; 3 ) discuss the privacy and security implications of the device.

\section{BACKGROUND}

\section{A. Video-Based Physiological Measurement}

Video-based photoplethysmography leverages camera sensors to detect blood flow via very subtle changes in light reflected from the skin (typically, but not exclusively, on the face). Over the past ten years iPPG has received a lot of attention from the research community. Advancements have been made in motion and ambient illumination tolerance meaning that iPPG now has practical applications [9].

Using a DSLR camera with a telephoto lens it is even possible to recover the heart rate of a subject at a distance of $50 \mathrm{~m} \mathrm{[10].} \mathrm{Using} \mathrm{infra-red} \mathrm{cameras} \mathrm{one} \mathrm{can} \mathrm{reliably} \mathrm{measure}$ heart rates in low visual spectrum lighting conditions [11]. Even videos that have been compressed using lossy spatial and temporal algorithms, such as TV recordings, can be used to recover the heart rate of a subject [12].

\section{B. Camouflage Against Vision Systems}

Video surveillance has become an acute problem, with advanced computer vision (CV) algorithms for identity recognition now in use in many places. Several solutions have been proposed to help confuse face detection and recognition systems [13]. For a design exploration of this domain see [14]. CV Dazzle [15] and Privacy Visor are two systems [16] that have been reduced to practice.

CV Dazzle and other systems spurred valuable debate about how one might opt-out of being surveilled. However, 


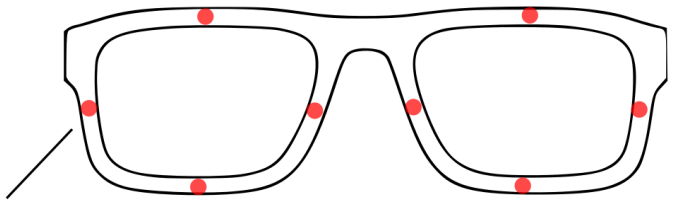

Infra-red LEDs on the inside face of the glasses frame illumnate the skin on the wearer's face. The eyes are shielded to prevent extended periods of exposure to IR light.

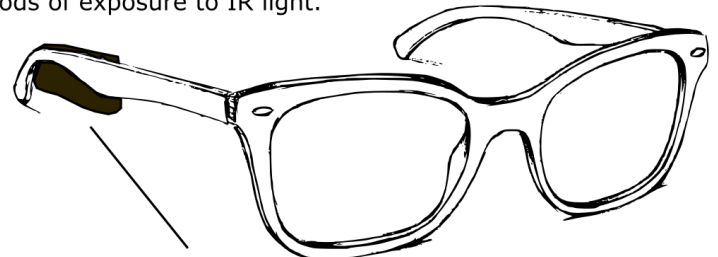

Battery and microcontroller mounted on the arm of the glasses or in the wearer's pocket.
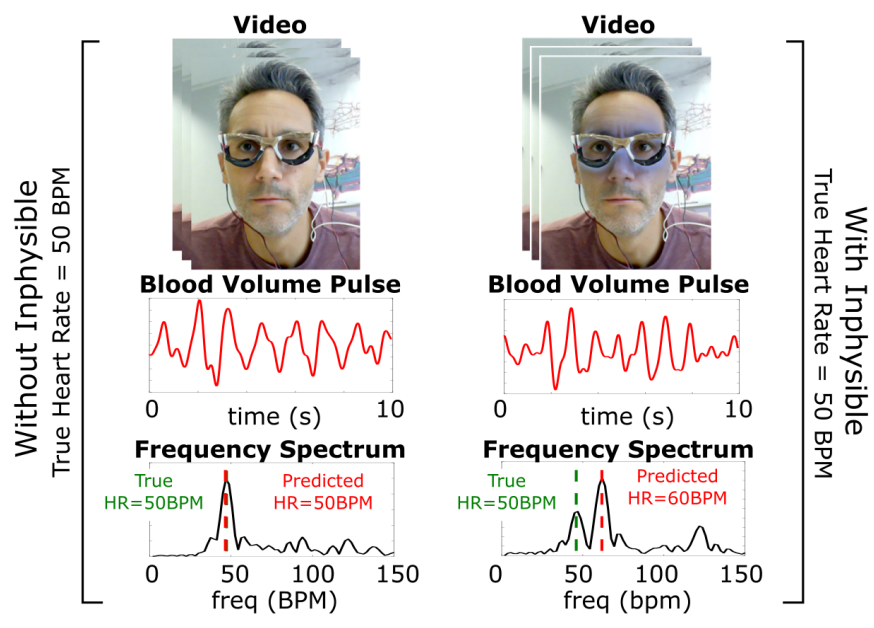

Fig. 1. InPhysible: A camouflage system that protects the wearer from measurement by video-based physiological monitoring systems. Note: The blue color on the face image is just for illustration - the IR LEDs are not visible to the unaided eye.
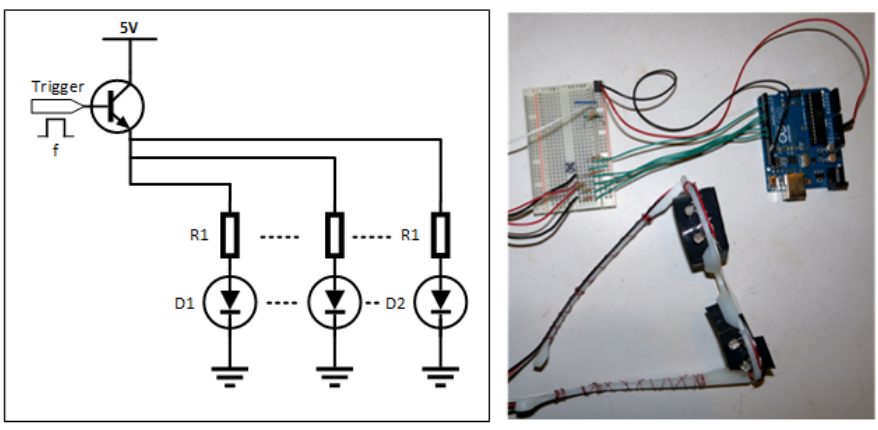

Fig. 2. Electronic schema with the triggered LEDs and our initial prototype of the 3D printed glasses.

these systems are impractical in many contexts as they obscure large parts of the face and make it obvious that the person is trying to evade face detection systems, this could be stigmatizing. Furthermore, iPPG requires only that skin can be detected and does not require the whole face to be visible. CV Dazzle could not prevent the PPG signal from being measured unless a large proportion of the skin area was covered by make-up or occluded by hair. BlindSpot [17] is a clever system created to prevent the recording of images or videos from CCD or CMOS cameras. The system uses automated tracking to identify the position of cameras and and then direct a pulsing light to distort the recorded images. However, there are occasions in which someone might not want to prevent video or image capture entirely, but avoid physiological measurement. BlindSpot does not allow for this. Furthermore, it requires a complex hardware set-up.

We present an active physiological signal spoofing method that not only prevents the pulse signal and vital signs from being measured but allows the user to spoof a false heart rate of their choosing. While the glasses in their current prototype form are not very subtle, due to the 3-D printed design, they could easily be adapted to be much less conspicuous.

\section{INPHYSIBLE}

In this section we detail the software and hardware implementation of our system. The design of such a system is not limited to the one we present; however, what we propose is a simple, low-cost and practical solution.

Hardware: InPhysible consists of a customized pair of glasses that have infra-red light emitting diodes (LEDs) embedded into the frame (see Figure 2). The frame of the glasses was 3D printed which makes the design highly customizable and allows an easy integration of holes to place LEDs. Eight infra-red LEDs were embedded into the frame on the side closest to the face. The light emitted by IR LEDs is outside the visible spectrum. Therefore, neither the bulbs nor the emitted light were noticeable to the wearer or an observer. While the skin was illuminated with the IR light the wearer's eyes were shielded from exposure by a rim around the glasses. Furthermore, the LEDs pointed away from the eyes in all locations. Despite these design choices further experimentation would be needed before the glasses could be worn for long periods of time.

The LEDs were connected to a micro-controller via wires. In this paper, we present our prototype to validate the feasibility of interfering with the measurement, and spoofing, of a user's heat rate. Our integration does not provide the most compact design. Our micro-controller (Arduino) was too big to be integrated in the glasses and was located in the user's pocket. An existing micro-controller or a reduced set of electronic components could easily be embedded in the glasses. For example, the electronics could be mounted on the frame behind the ear as illustrated in Figure 1.

Software: In order to test different camouflage strategies we developed software to control the brightness of the LEDs. The software was developed using the Arduino language and uses standard syntax to simply activate or inhibit the LEDs at a given time. During our initial testing we controlled the LED intensity to fit a sine wave profile:

$$
f(t)=1+\sin (2 \pi t) .
$$

We found that a simpler square wave profile could also provide similar results. Therefore, for simplicity we used a square wave in the final version. It is possible that the combination of the LED transfer function (signal current, emitted 


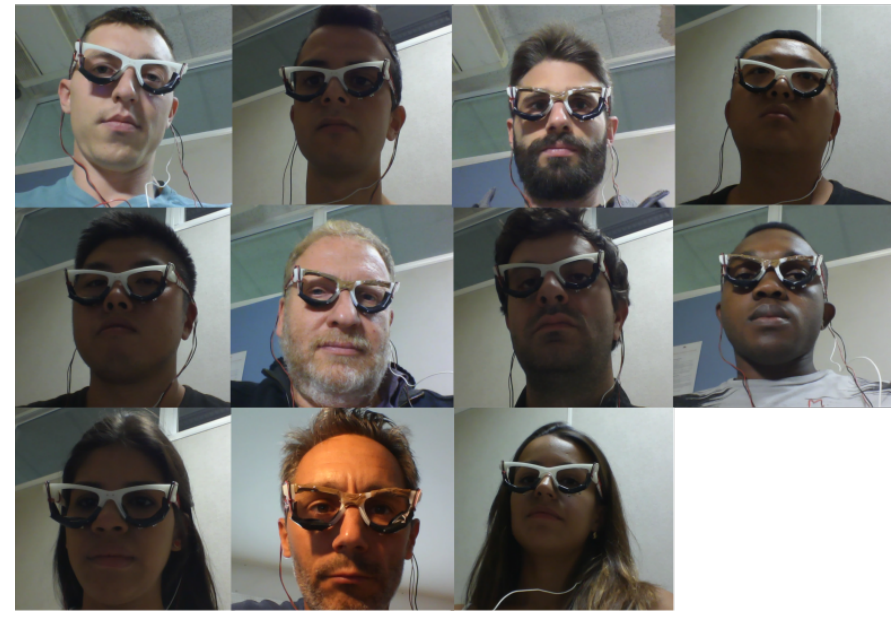

Fig. 3. Example frames from the videos collected in our user study. We tested the system in a range of ambient illumination conditions and recruited participants of various skin tones and ages.

intensity) and the skin infra-red absorption profile explain why a square wave may be sufficient. Our implementation is flexible enough to generate any kind of waveform and to fully control the frequency.

\section{USER STUDY}

To test the effectiveness of InPhysible at obstructing imaging PPG measurements we performed a validation study. We recruited 11 participants (two females, nine males; mean age: 30 years, st. dev.: 10 years) from a range of ethnicities (Caucasian, Black African, Asian). Table I shows the gender, ages and resting heart rates of the participants. Figure 3 shows example frames from the videos collected in our user study. We intentionally collected videos under a range of ambient illumination conditions. For each participant we recorded a six minute video while they were wearing the InPhysible glasses. The device used for recording was the Widescreen HD (720p) camera integrated in a Dell XPS 15 laptop. The videos were recorded at 30 frames-per-second (fps) and stored in MP4 format. Gold-standard measurements of the participants resting heart rate during the six minute trial were taken with a wristworn contact PPG sensor. We collected data while the subjects were stationary and under static ambient illumination. Dynamic illumination or head motions are only likely to make accurate HRs more difficult to recover and InPhysible more effective.

The six minute recording comprised of six one-minute "tasks". In which the interference pattern was varied:

Task 1: The system LEDs were off.

Task 2: The system generated a $1 \mathrm{~Hz}$ (60 BPM) pulse.

Task 3: The system generated a $1.5 \mathrm{~Hz}$ (90 BPM) pulse.

Task 4: The system generated a $2 \mathrm{~Hz}$ (120 BPM) pulse.

Task 5: The system generated pulses at randomly varying intervals (between 1-2 Hz [60 - 120 BPM]).

Task 6: The system generated a chirp with frequency increasing at a rate of $1 / 60 \mathrm{~Hz}$ per second from 1 to $2 \mathrm{~Hz}$.

\section{IMAging PhotoplethysmogRAPHy Method}

We implemented a commonly used approach for iPPG to test how the recovered heart rate was affected by interference from InPhysible. The method involves three main steps: 1) face detection and segmentation, 2) blood volume pulse recovery, 3) frequency analysis. We summarize our approach below but for much more detailed information refer to prior work using similar methods [3], [18], [19].

Face Detection and Segmentation: We used the OpenFace toolbox [20] for detecting the face within the video frames and localizing 32 facial feature points. A skin region of interest was segmented around the edge of the face. We did not try to optimize the region of interest. However, prior work has shown that this can help improve the accuracy of remote physiological measurements somewhat [21]. Nevertheless, we did note that the LED IR illumination affected most of the face and segmenting regions without IR interference would be difficult either manually or automatically.

Blood Volume Pulse Recovery: We implemented a commonly used source signal separation technique to recover the blood volume pulse (BVP) from the camera signals [3], [18], [19]. Spatially averaged pixel color signals from the face over a time window were computed for the red, green and blue color channels. Independent Component Analysis (ICA) was used to demix the signals and return three candidate source signals. Finally, the source signal with the highest peak power in the frequency range 45 - 150 BPM was selected as the estimated blood volume pulse.

Frequency Analysis: To determine the HR of the individual the BVP was first filtered using a zero-phase, 3rd order Butterworth bandpass filter with $3 \mathrm{~dB}$ cut-offs at 45 and 150 BPM. We then applied an FFT to the filtered BVP signal and selected the dominant (highest power) frequency peak in the range 45 - $150 \mathrm{BPM}$, this became our HR estimate.

\section{RESUlTs}

Figure 4 shows results for three participants in our user study (P1 - top, P2 - middle, P4 - bottom). The spectrogram plots show the normalized power in the frequency domain (black being the maximum (one) and white being the minimum (zero)). These plots were created with 15 -second time windows and a one-second step size. Thus, there is a 15second period at the beginning of each video before we have our first power spectrum estimate. We have highlighted each participant's resting heart rate in the plots. It is immediately obvious that the iPPG algorithm can detect their heart rate when the InPhysible system is not running. This is also reflected in the average error between the recovered $\mathrm{HR}$ and the gold-standard measurements in Table I. For Task 1 (no interference) the mean absolute error is $2.5 \mathrm{BPM}$ ( $4 \%$ error).

For all other tasks the interference from the system impacts the accuracy of the recovered heart rates. The interference patterns we tested have different properties. The constant frequency interference (at 60, 90 and $120 \mathrm{BPM}$ ) results in a high power dominant peak in the frequency domain (as seen in Figures 4 and 5). The patterns lead to the worst accuracy in terms of HR estimation. The mean absolute error with 60 

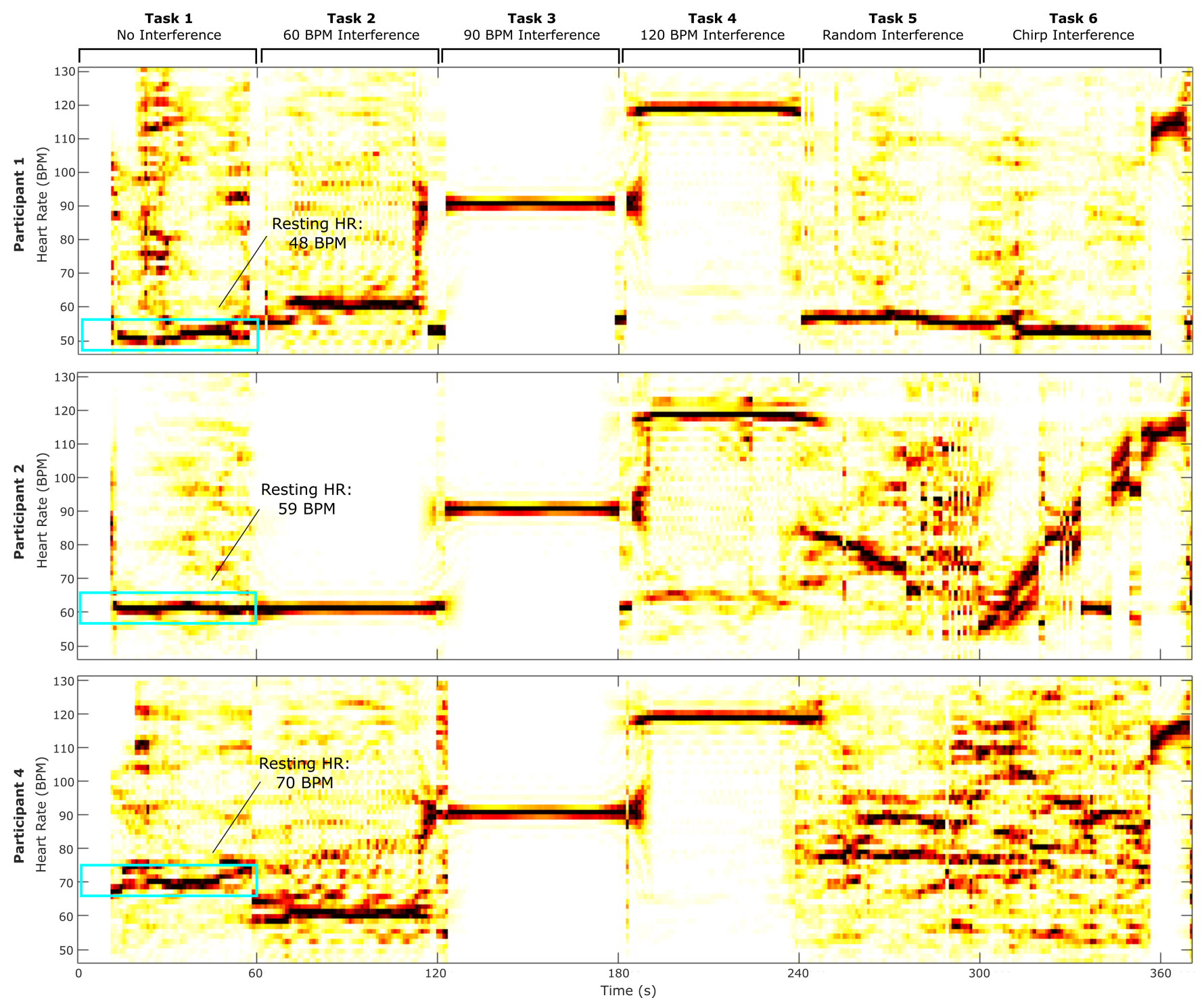

Fig. 4. Results from our user study. The spectrograms show unit normalized power in the frequency domain across time (black being the maximum (one) and white being the minimum (zero)). Top) Recovered physiological measurements from a participant with resting heart rate at 48 BPM. Middle) Recovered physiological measurements from a participant with resting heart rate at 60 BPM. Bottom) Recovered physiological measurements from a participant with resting heart rate at 70 BPM. In both cases the actual resting heart rate measured by a gold-standard contact sensor is highlighted and can be clearly seen when InPhysible is not emitting IR light. However, with InPhysible running the true heart rate is obscured.

BPM $(1 \mathrm{~Hz})$ interference is 14 BPM $(21 \%$ error), with 90 BPM $(1.5 \mathrm{~Hz})$ interference is $24 \mathrm{BPM}(36 \%$ error) and with $120 \mathrm{BPM}(2 \mathrm{~Hz})$ interference is $48 \mathrm{BPM}$ ( $72 \%$ error). Of these three fixed frequency patterns the 60 BPM interference results in the lowest mean absolute error simply because most people have a resting heart rate close to $60 \mathrm{BPM}$.

The random frequency and chirp signals create noise spread across the power spectrum as expected since there is noise at a range of frequencies in each time window. To illustrate this we have plotted the power spectra for two participants during each of the six tasks (Fig. 5). The true HR, as measured by the gold-standard device, is shown by the broken red line. The random frequency and chirp patterns are less effective at creating large mean absolute errors in the HR predictions. The HR peak is sometimes still identifiable in the frequency domain as the interference signal power is spread across a range of frequencies. The mean absolute error in the HR estimation was 6 BPM $(9 \%$ error) and 10 BPM (15\% error) for the random frequency and chirp interference patterns respectively. However, the results show that all the interference patterns create errors in the HR estimates. Average errors of $10 \mathrm{BPM}$ or larger render iPPG measurements more or less useless for practical applications.

\section{DISCUSSION}

\section{A. Optimal Interference Patterns}

One can easily notice from the plots in Figure 4 that the heart rate of an individual naturally fluctuates very subtly over time, this is known as heart rate variability. Therefore, if the interference from the system were at a 

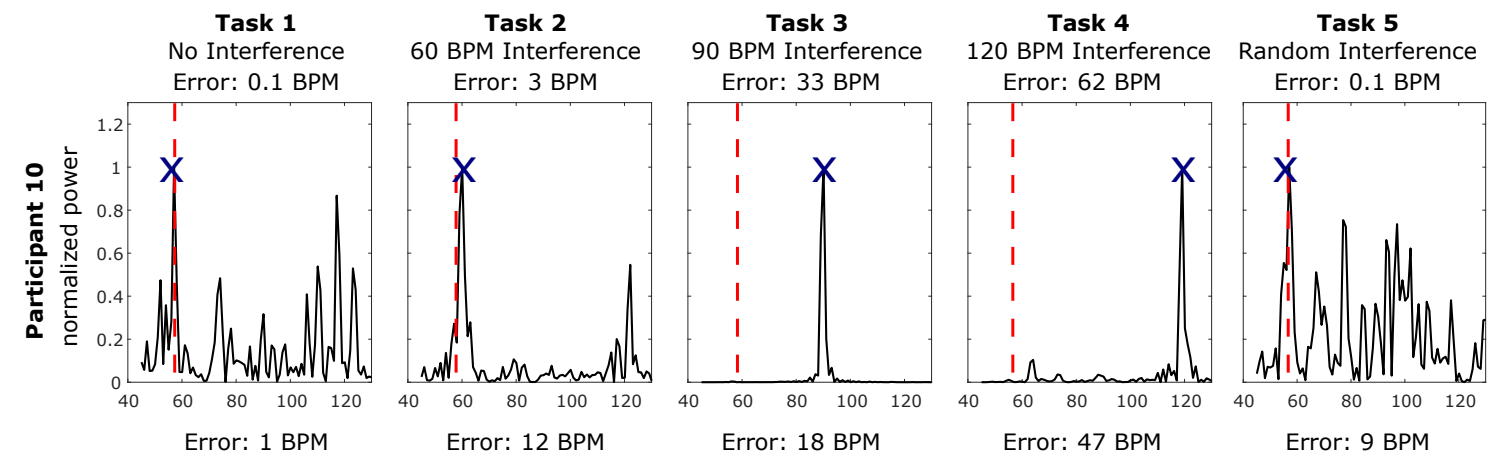

Task 6
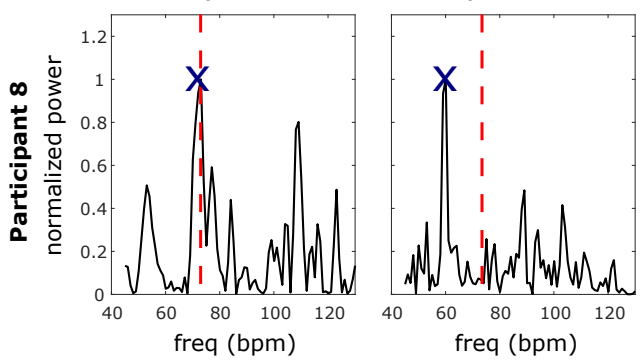

Error: 18 BPM
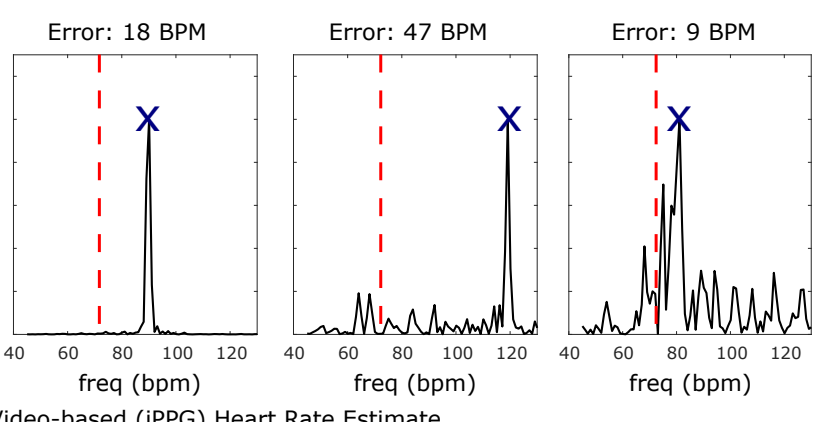

Chirp Interference

Error: 35 BPM

- _ Gold-standard (contact) HR Measurement $X$ Video-based (iPPG) Heart Rate Estimate

Fig. 5. The recovered pulse wave power spectra for two participants during each of the six tasks. The gold-standard heart rate is reflected by the broken red line. Top row) Participant 10 had a resting HR of 57 BPM. Bottom row) Participant 8 had a resting HR of 72 BPM. The peak selected by the camera-based (iPPG) algorithm is shown by a blue cross. For Task 1 (no interference) the HR estimates are very accurate; however, for all other tasks errors exist. The random interference was the least effective at obscuring the HR and the static frequency pulses at (60, 90 and 120 BPM) were the most effective.

TABLE I

The Mean Absolute Error (in Beats-Per-Minute) Between iPPG Measurements and Gold-Standard Contact Heart Rate for the Different InPhysible Interference Patterns. The Gold-standard Measurement of the Average Resting Heart Rate is Shown at the Top Alongside the Participant Demographic Information.

\begin{tabular}{|c|c|c|c|c|c|c|c|c|c|c|c|c|c|}
\hline \multirow[b]{5}{*}{ Task } & Participants & P1 & $\mathrm{P} 2$ & P3 & P4 & P5 & P6 & P7 & P8 & P9 & P10 & P11 & Mean (std) \\
\hline & Gender & M & M & M & M & M & M & M & $\mathrm{F}$ & M & M & $\mathrm{F}$ & \\
\hline & Age & 30 & 26 & 55 & 33 & 42 & 22 & 31 & 20 & 25 & 24 & 23 & $30.1(10.3)$ \\
\hline & Resting HR (BPM) & 48 & 59 & 79 & 70 & 54 & 67 & 67 & 72 & 68 & 57 & 84 & $66.0(10.9)$ \\
\hline & Interference & \multicolumn{12}{|c|}{ HR Estimation Mean Absolute Error } \\
\hline 1 & None & 0.05 & 0.94 & 1.09 & 2.93 & 0.06 & 3.08 & 7.08 & 1.08 & 5.93 & 0.06 & 4.91 & $2.47(2.52)$ \\
\hline 2 & $60 \mathrm{BPM}$ & 7.06 & 1.07 & 18.93 & 7.93 & 51.11 & 6.93 & 6.93 & 11.93 & 8.93 & 3.07 & 24.93 & $13.53(14.21)$ \\
\hline 3 & 90 BPM & 42.10 & 31.10 & 11.10 & 20.10 & 36.10 & 23.10 & 23.10 & 18.10 & 22.10 & 33.10 & 5.10 & $24.10(10.89)$ \\
\hline 4 & $120 \mathrm{BPM}$ & 71.13 & 60.13 & 40.13 & 49.13 & 4.95 & 52.13 & 52.13 & 47.13 & 51.13 & 62.13 & 34.13 & $47.57(17.40)$ \\
\hline 5 & Random Freq. & 7.06 & 16.08 & 0.91 & 7.09 & 3.06 & 1.08 & 3.93 & 9.09 & 1.08 & 0.06 & 19.11 & $6.23(6.38)$ \\
\hline 6 & Chirp Noise & 4.06 & 2.94 & 3.92 & 13.09 & 15.08 & 7.08 & 0.07 & 5.93 & 2.08 & 35.10 & 14.92 & $9.50(9.98)$ \\
\hline
\end{tabular}

constant frequency it might be easy to detect and filter out. Adding small variations in the LED pulse intervals for successive pulses would result in a more robust system. These dynamics could easily be learned from past human pulse signal recordings. Although, small random variations in frequencies may be sufficient. Our results also show that the spread of frequencies in the HR signal should not be too large, otherwise they may fail to obscure the genuine pulse signal. Looking at the results in Figure 5 for P8 it seems that both the random and chirp interference patterns fail to obscure the dominant heart rate signal (observed close to $70 \mathrm{BPM})$. It is reasonable that these patterns may not be an optimal for HR interference as they add low power noise across a broad range of frequencies and thus the heart rate peak is still visible. This result is reflected in the mean absolute errors in Table I.

\section{B. Technical Considerations}

There are some technical limitations of our system that can hinder its effectiveness as a camouflage. In strong ambient lighting conditions the LEDs we used would not be bright enough to cover the natural change in color of the user's skin. More LEDs could be placed around the glasses; however, this would result in higher energy consumption.

One advantage of our system is the use of IR light. The interference is captured by a camera and our system can operate as a camouflage. In addition, the change in color of the skin due to the LEDs is not perceptible to the human eye. However, a human observer could detect the change of luminosity around the wearers' eyes when looking at images captured by a camera. We could use LEDs of differing light frequencies, adapting to the wearer's skin tone to make this signal harder to detect. This may be complex to implement 
and highly sensitive to the environmental lighting conditions.

During our investigations, our system gave good results across a range of personal attributes (including gender, skin tone and age). However, we do know that pulse detection is more difficult for older people and those wearing make-up. InPhysible may offer less benefit to certain demographics.

\section{Privacy and Ethics}

With the number of cameras in public spaces increasing and the number of devices in the home with visual sensors connected to the Internet there is increasing concern about surveillance. Systems that can measure personal information threaten privacy and security. HR and HRV measurements could be used to understand reactions to advertisements or as part of security screening at airports or in other public spaces. The issue is not so much that one can capture this data without someone knowing, but rather that that information can be used for other purposes. As long as there is no way to fully control how such data can be used, our system provides a simple valuable attempt to prevent unwanted physiological measurement. During job interviews, poker games, political debates or any other high-stakes situation, monitoring a person's HRV could provide information regarding their stress level. With our system the wearer could provide false information or interference in these measurements.

In many cases capturing physiological information remotely is beneficial for the subject. For example, it may not be possible to attach contact physiological sensors to a person with delicate skin. In this case the user could simply switch off the system. An external observer would find it difficult to determine if the system was running or not. Further discussion and the implications for health, security and privacy is a topic which goes beyond the scope of this paper. However, we have demonstrated that InPhysible effectively prevents the measurement of the wearer's heart rate using video-based methods.

\section{CONCLUSIONS}

We have presented InPhysible, the first camouflage device against video-based physiological measurement. The infrared system can be embedded into any pair of glasses and disrupts the measurement of the iPPG signal while being imperceptible to the human eye. The system could be extended to other pieces of head wear (e.g., a scarf or hat).

We evaluated our system on 11 people with different skin tones and tested a number of interference patterns. Our evaluation validated the efficacy of our system and has informed future design of interference patterns. We discussed the technical challenges and learnings from our prototype solution and the trade-off between privacy and security. In future, we plan to investigate others physiological camouflage systems. Our goal is not to provide systems to jeopardize vital sign retrieval, but to maintain users' control over their personal data.

\section{REFERENCES}

[1] W. Verkruysse, L. O. Svaasand, and J. S. Nelson, "Remote plethysmographic imaging using ambient light," Optics express, vol. 16, no. 26, pp. $21434-21445,2008$.
[2] M.-Z. Poh, D. McDuff, and R. W. Picard, "Advancements in noncontact, multiparameter physiological measurements using a webcam," IEEE Transactions on Biomedical Engineering, vol. 58, no. 1, pp. 7-11, 2011.

[3] — , "Non-contact, automated cardiac pulse measurements using video imaging and blind source separation," Optics Express, vol. 18, no. 10, pp. 10762-10774, 2010.

[4] S. Fernando, W. Wang, I. Kirenko, G. de Haan, S. Bambang Oetomo, H. Corporaal, and J. van Dalfsen, "Feasibility of contactless pulse rate monitoring of neonates using google glass," in Proceedings of the 5th EAI International Conference on Wireless Mobile Communication and Healthcare. ICST (Institute for Computer Sciences, SocialInformatics and Telecommunications Engineering), 2015, pp. 198201.

[5] C. Hurter and D. McDuff, "Cardiolens: remote physiological monitoring in a mixed reality environment," in ACM SIGGRAPH 2017 Emerging Technologies. ACM, 2017, p. 6.

[6] D. McDuff, C. Hurter, and M. Gonzalez-Franco, "Pulse and vital sign measurement in mixed reality using a hololens," in Proceedings of the 23rd ACM Symposium on Virtual Reality Software and Technology. ACM, 2017, p. 34.

[7] L. Tarassenko, M. Villarroel, A. Guazzi, J. Jorge, D. Clifton, and C. Pugh, "Non-contact video-based vital sign monitoring using ambient light and auto-regressive models," Physiological measurement, vol. 35, no. 5, p. 807, 2014.

[8] H.-Y. Wu, M. Rubinstein, E. Shih, J. V. Guttag, F. Durand, and W. T. Freeman, "Eulerian video magnification for revealing subtle changes in the world." ACM Trans. Graph., vol. 31, no. 4, p. 65, 2012.

[9] D. McDuff, J. R. Estepp, A. M. Piasecki, and E. B. Blackford, "A survey of remote optical photoplethysmographic imaging methods," in Engineering in Medicine and Biology Society (EMBC), 2015 37th Annual International Conference of the IEEE. IEEE, 2015, pp. 63986404.

[10] E. B. Blackford, J. R. Estepp, A. M. Piasecki, M. A. Bowers, and S. L. Klosterman, "Long-range non-contact imaging photoplethysmography: Cardiac pulse wave sensing at a distance," in Proc. of SPIE Vol, vol. 9715, 2016, pp. 971 512-1.

[11] M. van Gastel, S. Stuijk, and G. de Haan, "Motion robust remote-ppg in infrared," IEEE Transactions on Biomedical Engineering, vol. 62, no. 5, pp. 1425-1433, 2015.

[12] D. McDuff, E. B. Blackford, and J. R. Estepp, "The impact of video compression on remote cardiac pulse measurement using imaging photoplethysmography," in Automatic Face \& Gesture Recognition (FG 2017), 2017 12th IEEE International Conference on. IEEE, 2017, pp. 63-70.

[13] M. J. Wilber, V. Shmatikov, and S. Belongie, "Can we still avoid automatic face detection?" in Applications of Computer Vision (WACV), 2016 IEEE Winter Conference on. IEEE, 2016, pp. 1-9.

[14] K. Krombholz, A. Dabrowski, M. Smith, and E. Weippl, "Exploring design directions for wearable privacy," 2017.

[15] A. Harvey, "Cv dazzle: Camouflage from computer vision," New York University, Tech. Rep., 2012.

[16] T. Yamada, S. Gohshi, and I. Echizen, "Privacy visor: Method for preventing face image detection by using differences in human and device sensitivity," in IFIP International Conference on Communications and Multimedia Security. Springer, 2013, pp. 152-161.

[17] S. N. Patel, J. W. Summet, and K. N. Truong, "Blindspot: Creating capture-resistant spaces," in Protecting Privacy in Video Surveillance. Springer, 2009, pp. 185-201.

[18] D. McDuff, S. Gontarek, and R. W. Picard, "Improvements in remote cardiopulmonary measurement using a five band digital camera," IEEE Transactions on Biomedical Engineering, vol. 61, no. 10, pp. 25932601, 2014.

[19] E. B. Blackford and J. R. Estepp, "Effects of frame rate and image resolution on pulse rate measured using multiple camera imaging photoplethysmography," in SPIE Medical Imaging. International Society for Optics and Photonics, 2015, pp. 94 172D-94 172D.

[20] T. Baltrušaitis, P. Robinson, and L.-P. Morency, "Openface: an open source facial behavior analysis toolkit," in Applications of Computer Vision (WACV), 2016 IEEE Winter Conference on. IEEE, 2016, pp. $1-10$.

[21] G. Lempe, S. Zaunseder, T. Wirthgen, S. Zipser, and H. Malberg, "Roi selection for remote photoplethysmography." in Bildverarbeitung für die Medizin. Springer, 2013, pp. 99-103. 